\title{
Polimorfismos del TLR4 en pacientes con enfermedad de Stargardt: un estudio familiar
}

\section{Toll-like receptor 4 polymorphisms in patients with Stargardt disease: A family study}

\author{
Ramses Rosales-Diaz'1,2, Alejandro González-de la Rosa ${ }^{3,4}$, Nicole Macriz-Romero ${ }^{4}$, \\ Juan C. Altamirano-Vallejo ${ }^{3,4}$, Abril B. Martinez-Rizo ${ }^{1}$, Arturo Santos-Garcia ${ }^{3,4}$, \\ Adolfo D. Rodriguez-Carrizalez ${ }^{2}$ y Jose Navarro-Partida ${ }^{3,4 *}$ \\ ${ }^{1}$ Unidad Académica de Medicina, Universidad Autónoma de Nayarit, Tepic, Nay.; ${ }^{2}$ Instituto de Terapéutica Experimental y Clínica, Universidad de

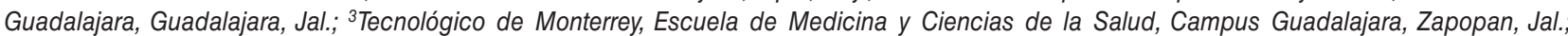 \\ ${ }^{4}$ Centro de Retina Médica y Quirúrgica S.C., Centro Médico Puerta de Hierro, Zapopan, Jal. México
}

\section{Resumen}

Objetivo: Las células del epitelio pigmentario retiniano tienden a la apoptosis en la distrofia macular de Stargardt (STGD). Anteriormente, los polimorfismos de un solo nucleótido (SNP) del gen del receptor Toll de tipo 4 (TLR4) se habían relacionado con la apoptosis y la inflamación. Por tanto, este estudio se realizó para investigar si los SNP del TLR4 están asociados con la STGD en un estudio familiar. Métodos: Se incluyeron 4 pacientes mexicanos consanguíneos con diagnóstico clínico de STGD (4 mujeres) y 12 familiares no afectados. Se utilizaron como control 109 sujetos (40 hombres y 69 mujeres; edad, $63.28 \pm 7.93$ años) sin enfermedades maculares ni antecedentes familiares. Los SNP rs4986790, rs1927911, rs12377632, rs2149356 y rs11536889 del gen TLR4 fueron genotipados usando un ensayo de discriminación alélica Taqman ${ }^{\circledR}$. Resultados: La frecuencia del alelo menor rs4986790 (G) fue significativamente mayor en pacientes con STGD en comparación con los controles (25 vs. 1\%; $p=0.0012)$. El genotipo portador del alelo menor rs4986790 (AG) fue más frecuente en los pacientes con STGD (50\%) en comparación con sus familiares y controles (8 y 2.75\%, respectivamente) de manera significativa $(p=0.0048)$. Las frecuencias alélicas y genotípicas de los SNP restantes no fueron significativas entre pacientes con STGD y controles ( $p>0.5)$. Los familiares no afectados mostraron frecuencias alélicas y genotípicas similares a las encontradas en los controles. Conclusión: El alelo menor del SNP rs4986790 (G) y el genotipo portador (AG) se relacionaron con la enfermedad de Stargardt clínica. Curiosamente, el rs4986790 se ha descrito como un promotor de la apoptosis. Así, pues, este polimorfismo del gen TLR4 debe considerarse como un marcador en estudios futuros.

Palabras clave: Enfermedad de Stargardt. Receptor tipo toll 4. Polimorfismos. Estudio genético familiar.

\section{Abstract}

Purpose: Retinal pigment epithelial cells exhibit a propensity for apoptosis in Stargardt macular dystrophy (STGD). Previously, single-nucleotide polymorphisms (SNPS) in the toll-like receptor 4 (TLR4) gene have been related to apoptosis and inflammatory response. Therefore, this study was undertaken to investigate whether TLR4 SNPs are associated with STGD in a family-based

\footnotetext{
Correspondencia:

*Jose Navarro-Partida

Edif. de Ciencias de la Salud

Tecnológico de Monterrey, Campus Guadalajara

Av. General Ramón Corona, 2514

Fecha de recepción: 02-05-2019

Fecha de aceptación: 05-09-2019

E-mail: josenavarro@tec.mx

DOI: 10.24875/RMO.M20000102

Disponible en internet: 02-01-2020

Rev Mex Oftalmol. 2020;94(1):9-15

www.rmo.com.mx 0187-4519/C 2019 Sociedad Mexicana de Oftalmología. Publicado por Permanyer. Éste es un artículo open access bajo la licencia CC BY-NC-ND (http://creativecommons.org/licenses/by-nc-nd/4.0/).
} 
study. Methods: Four blood-related Mexican patients with a clinical diagnosis of STGD (4 women) and 12 of their unaffected relatives were included in the study. A total of 109 subjects (40 men and 69 women; age, $63.28 \pm 7.93$ years) without macular affections, family history, or inherited macular dystrophies were used as controls. SNPs rs4986790, rs 1927911, rs12377632, rs2149356, and rs 11536889 of the TLR4 gene were genotyped using a Taqman ${ }^{\circledR}$ Allelic Discrimination Assay. Results: The frequency of the minor allele of rs 4986790 (G) was significantly higher in STGD patients compared to control subjects (25\% vs. $1 \%, p=0.0012)$. The genotype carrying the minor allele of rs 4986790 (AG) was more frequent in STGD patients (50\%) compared with their relatives and unrelated control subjects (8 and $2.75 \%$, respectively), with statistical significance $(p=0.0048)$. The allele and genotype frequencies of the remaining SNPS were not significant between STGD patients and control subjects $(p>0.5)$. Unaffected relatives of STGD patients showed allele and genotype frequencies similar to those observed in control subjects. Conclusion: Minor alleles of the SNPs rs4986790 (G) and genotypes carrying it (AG) were related to clinical STGD in one family. Interestingly, rs 4986790 has been described as a promoter of apoptosis. Therefore, this TLR4 gene polymorphism should be considered as a marker in future studies.

Key words: Stargardt disease. Toll-like receptor 4. Polymorphisms. Family-based genetic study.

\section{Introducción}

La enfermedad de Stargardt o distrofia macular de Stargardt (STGD) (Online Mendelian Inheritance in Man [OMIM]: 248200) es una forma autosómica recesiva de distrofia macular de inicio juvenil descrita por primera vez en 1909 por el oftalmólogo alemán Karl Stargardt ${ }^{1}$. Es el tipo más común de distrofia macular hereditaria recesiva, con una incidencia estimada de 1 en cada 10,000 nacidos vivos. Representa aproximadamente el $7 \%$ de todas las enfermedades de la retina, y se presenta típicamente en niños y adultos jóvenes ${ }^{2,3}$. El diagnóstico de STGD se basa clásicamente en los antecedentes familiares, la agudeza visual, el examen del fondo de ojo, la angiografía con fluoresceína, la autofluorescencia del fondo y la electrorretinografía ${ }^{4}$.

La forma típica de STGD (STGD1, OMIM \#248200) es causada por mutaciones en el gen del transportador de casete de unión a ATP 4 (ABCA4) ubicado en 1p22.1, con una transmisión autosómica recesiva homocigótica o heterocigótica compuesta. Las mutaciones del gen de la proteína 1 similar a la prominina (PROM1), ubicado en $4 p 15.32$, causan una forma autosómica dominante (STGD4, OMIM \#603786). Las mutaciones dominantes del gen de la elongasa 4 de ácidos grasos (ELOVL4) (6q14.1) causan distrofias maculares similares a Stargardt (STDG3, OMIM \#600110) 5 .

La patogénesis de la enfermedad de Stargardt asociada con mutaciones del gen ABCA4 ha sido bien establecida. ABCA4 participa en el ciclo retinoide como una «flipasa» que transporta el subproducto $\mathrm{N}$-retinilideno-fosfatidiletanolamina ( $\mathrm{N}$-retinilideno-PE) a través de las membranas del disco. Mediante este mecanismo, ABCA4 previene la acumulación de retinoides en el espacio subcelular después del fotoblanqueo de rodopsina ${ }^{5}$. La ausencia del transporte de N-retinilideno-PE a través de la membrana del disco conduce a su atrapamiento y reacción con todo-trans-retinal (atRAL) para formar di-retinoide-piridinio-fosfatidiletanolamina (A2PE). Cuando los fotorreceptores se renuevan, el A2PE se convierte en di-retinoide-piridinio-etanolamina (A2E), un dímero de la vitamina $A$, que queda atrapado permanentemente en el epitelio pigmentario de la retina (EPR) porque no puede hidrolizarse. El A2E es un componente importante de la lipofuscina, un sello distintivo de la degeneración celular ${ }^{5}$. El atRAL acumulado puede volver a asociarse con la opsina, y el complejo resultante es menos efectivo para activar la cascada visual que la rodopsina fotoactivada; esto puede explicar la prolongada adaptación a la oscuridad encontrada en pacientes con STGD5,6. La lipofuscina puede acumularse hasta 5 veces por encima de los valores normales, y el exceso de A2E en el EPR afecta su función y supervivencia porque actúa como un detergente que compromete la membrana celular y las funciones metabólicas. Además, en presencia de oxígeno y luz de longitud de onda azul, la lipofuscina forma radicales libres de epóxido que inducen la muerte de células del EPR $^{6}$. La muerte de las células del EPR conduce a la degeneración secundaria de fotorreceptores ${ }^{7}$.

La activación microglial por proteínas retinianas endógenas que surgen de fotorreceptores lesionados juega un papel importante en la patogénesis de la degeneración retiniana. En un estudio exhaustivo en un modelo de ratón de STGD (causado por la eliminación retardada de atRAL de la retina), se demuestra una contribución importante de la activación microglial mediada por el receptor Toll de tipo 4 (TLR4) en la inflamación de la retina, que agrava la muerte de las células de la retina ${ }^{8}$.

El gen del TLR4 ha sido asociado en varios informes con apoptosis de células retinianas. Por ejemplo, el TLR4 y su vía de señalización se han relacionado con la muerte de las células ganglionares de la retina $(R G C)^{9-11}$ y con 


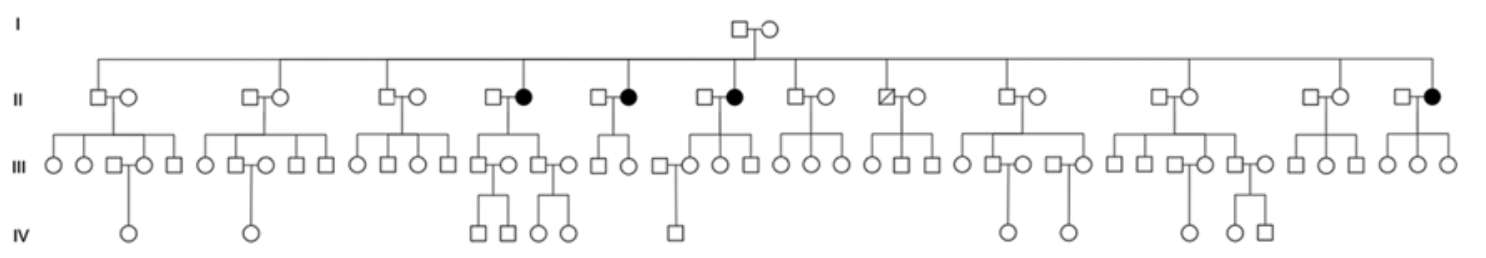

Figura 1. Pedigrí de la familia afectada por distrofia macular de Stargardt (STGD). Las personas afectadas se muestran en sombreado y los sujetos sanos se muestran como figuras abiertas. Los círculos representan mujeres y los cuadrados representan hombres. Cuatro hermanos en la segunda generación (II: 8, II: 9, II: 10 y II: 22) están afectados. Este patrón es compatible con la transmisión autosómica recesiva observada en la STGD.

la apoptosis de las RGC y las células endoteliales de la retina en modelos con alto contenido de glucosa que imitan el microambiente de la retinopatía diabética ${ }^{12,13}$.

El TLR4 es un miembro de la familia de receptores de reconocimiento de patrones que son una parte clave del sistema inmune innato. Se ha identificado en el sistema nervioso central y en la retina ${ }^{9}$. El gen del TLR4 está ubicado en el locus 9q33.1 que abarca una región genómica de $\sim 13.3 \mathrm{~kb}$ con cuatro exones (Ensamblado; GRCh38.p2, RefSeqGene; NG_011475.1, ID de gen; TLR4 7099) ${ }^{14}$ que codifica un receptor de reconocimiento de patógenos transmembrana involucrado en la detección de lipopolisacáridos (LPS) de bacterias Gram negativas y otros ligandos exógenos o endógenos ${ }^{15}$. Este gen muestra varios polimorfismos de un solo nucleótido (SNP) que presumiblemente influyen en la actividad y la función de su producto ${ }^{16}$. Se ha demostrado que los polimorfismos de TLR4 en humanos pueden contribuir a la gravedad de las enfermedades degenerativas oculares como el glaucoma ${ }^{17,18}$ o la degeneración macular relacionada con la edad ${ }^{19}$.

Con base en estudios que sugieren la participación del TLR4 en la patogénesis de la enfermedad de Stargardt y la asociación de polimorfismos del TLR con trastornos degenerativos de la retina, decidimos evaluar la asociación de SNP en regiones codificantes y no codificantes del gen TLR4 (locus 9q32- q33) con la enfermedad de Stargardt macular en una familia con diagnóstico clínico de enfermedad de Stargardt. Sorprendentemente, esta marca genética ha sido descrita como proapoptótica en otros estudios.

\section{Pacientes y métodos}

\section{Pacientes}

Se obtuvo ADN de 4 pacientes afectados (4 mujeres) y 12 familiares no afectados de una familia mexicana multigeneracional. El pedigrí de la familia afectada se muestra en la figura 1. Se incluyeron un total de
109 (40 hombres y 69 mujeres; edad $63.28 \pm 7.93$ años) muestras de control de una población mexicana emparejada. Los pacientes diagnosticados con STGD se sometieron a un examen ocular completo, angiografía con fluoresceína y tomografía de coherencia óptica de alta definición (HD-OCT). Además, se realizó una electrorretinografía para corroborar el diagnóstico de STGD. La fotografía del fondo de ojo, la fotografía de fondo libre de rojo y las imágenes de HD-OCT de pacientes con STGD se presentan en la figura 2. Los sujetos control tenían una historia clínica completa, ninguno de ellos tenía antecedentes familiares de enfermedad macular, y fueron examinados cuidadosamente para descartar cualquier enfermedad ocular. Se obtuvo el consentimiento informado de todas las personas que participaron en el estudio. El estudio fue aprobado por el comité de ética local y cumplió las directrices de la declaración de Helsinki. Todos los detalles del estudio se explicaron a los pacientes y a los sujetos control antes de obtener el consentimiento informado para el cribado genético.

\section{Preparación de muestras y genotipado}

Los SNP objetivo se encuentran en regiones no codificantes del gen TLR4 (locus 9q32-q33); rs1927911 G/A, rs12377632 T/C y rs2149356 G/T dentro de los intrones, rs11536889 G/C dentro de la región 3' no traducida y rs4986790 A/G (Asp299Gly) ubicado en el tercer exón (variante de transcripción 1; RefSeq: NM_138554.3). La posición de los polimorfismos es 8595, 11271, 12740 , 16672 y 13843, respectivamente (Ensamblaje; GRCh38. p2, RefSeqGene; NG_011475.1, ID de gen; TLR4 7099).

El ADN genómico se extrajo de leucocitos de sangre periférica y se purificó con el kit para sangre de Qiagen QIAmp DNA (Qiagen, Valencia, CA, EE.UU.). El genotipado de los SNP objetivo se realizó utilizando ensayos de genotipado de SNP con TaqMan validados (TaqMan: Applied Biosystems, Inc. [ABI], Foster City, CA). Los SNP se amplificaron utilizando reacción en cadena de la 


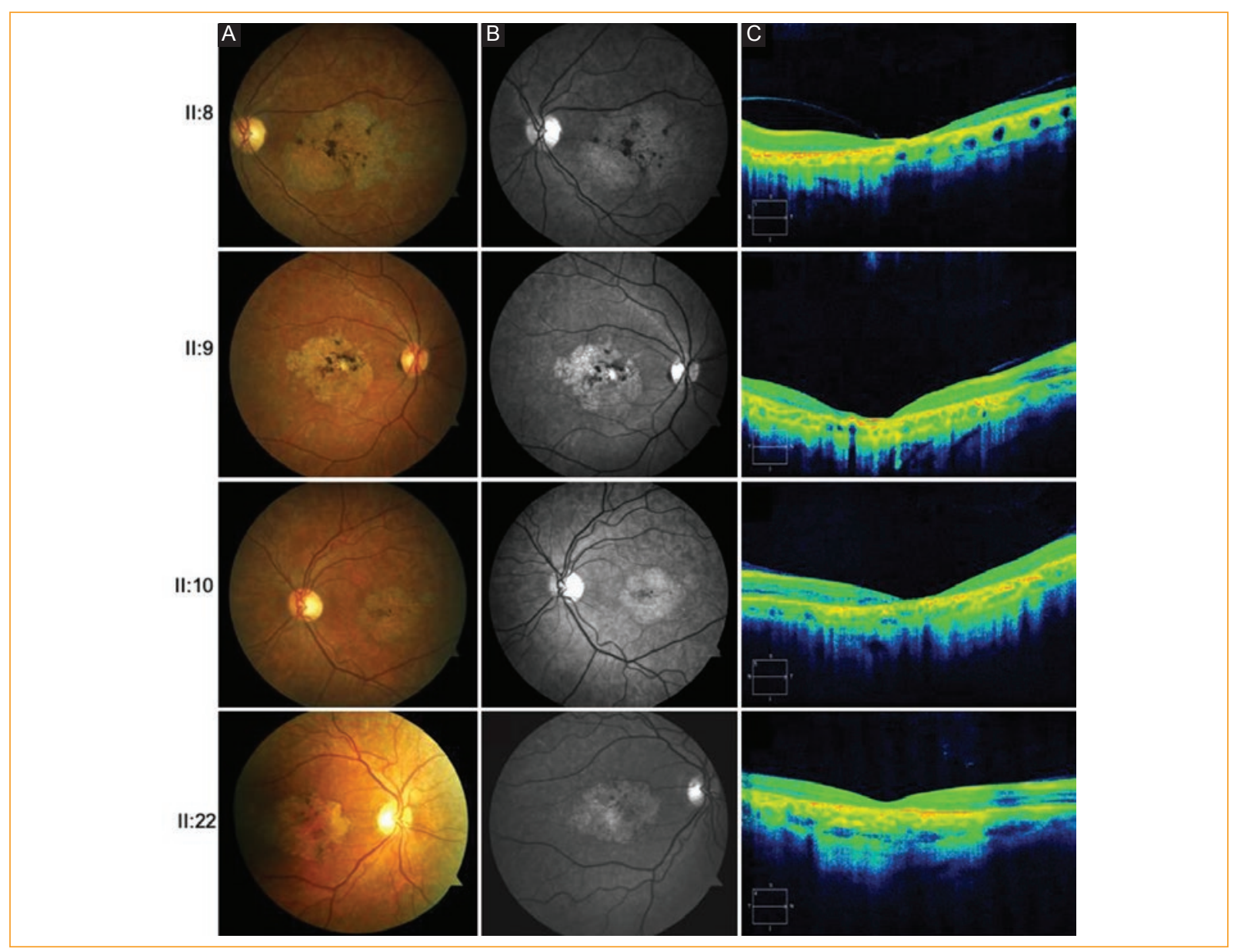

Figura 2. Imágenes representativas de los estudios diagnósticos de sujetos afectados. Fotografía de fondo (A), fotografía de fondo libre de rojo (B) e imágenes de tomografía de coherencia óptica macular de alta definición (C) de pacientes con enfermedad de Stargardt (STGD). Las filas 1, 2, 3 y 4 corresponden a imágenes de diagnóstico de II: 8, II: 9, II: 10 y II: 22, pacientes con STGD.

polimerasa (PCR) en tiempo real con $0.5 \mu \mathrm{M}$ de cebadores y Master Mix para genotipado de TaqMan (TaqMan: Applied Biosystems, Inc. [ABI], Foster City, CA) con $30 \mathrm{ng}$ de ADN templado en la mezcla de amplificación $(25 \mu \mathrm{l})$. La señal de fluorescencia de la sonda se detectó con el sistema de PCR en tiempo real StepOnePlus ${ }^{\mathrm{TM}}$ (Applied Biosystems, Inc. [ABI], Carlsbad, CA).

\section{Análisis estadístico}

Se utilizó el software estadístico IBM SPSS (versión 21.0, SPSS, Inc., Chicago, IL) para los análisis estadísticos. El equilibrio de Hardy-Weinberg se analizó utilizando frecuencias de genes obtenidas mediante un simple conteo de genes y la prueba Chi-cuadrada $\left(\mathrm{X}^{2}\right)$ bcon la corrección de Yates para comparar los valores observados y esperados. Las diferencias en las frecuencias de genotipo entre los casos y los controles se analizaron mediante la prueba exacta de Fisher o $\mathrm{X}^{2}$ dependiendo de los recuentos celulares. Los haplotipos inferidos y el LD (desequilibrio del ligamiento) cuantificados entre los loci bialélicos se estimaron utilizando el software en línea SNPStats (http://bioinfo.iconologia. net/SNPStats). La importancia de una asociación se determinó mediante análisis de tablas de contingencia utilizando pruebas de $\mathrm{X}^{2}$ o prueba exacta de Fisher. La significancia estadística se definió como una $p<0.05$.

\section{Resultados}

\section{Frecuencias de alelos y genotipos}

Las frecuencias alélicas de SNP en pacientes con STGD, sus familiares y los sujetos control se muestran en la tabla 1. Los SNP rs1927911 G/A, rs12377632 T/C, rs2149356 G/T, rs11536889 G/C y rs4986790 A/G se genotipificaron en todos los sujetos y se encontraban 
Tabla 1. Frecuencia de alelos SNP en los grupos de STGD y control

\begin{tabular}{|ll|c|c|c|c|c|}
\hline SNP/alelos & & STGD & Familiares & Control & $p$ \\
\cline { 3 - 7 } & & $n=8^{*}$ & $n=24$ & $n=218^{*}$ & caso vs, control & general \\
\hline rs4986790 & A/G & $2(0.25)$ & $1(0.04)$ & $3(0.01)$ & 0.00120 & 0.00772 \\
\hline rs1927911 & G/A & $2(0.25)$ & $6(0.25)$ & $56(0.26)$ & 0.71229 & 0.99651 \\
\hline rs12377632 & T/C & $3(0.375)$ & $5(0.21)$ & $105(0.48)$ & 0.81624 & 0.06467 \\
\hline rs2149356 & G/T & $4(0.5)$ & $7(0.29)$ & $60(0.28)$ & 0.32393 & 0.38194 \\
\hline rs11536889 & G/C & $0(0)$ & $0(0)$ & $28(0.13)$ & 0.59151 & 0.26700 \\
\hline
\end{tabular}

*Frecuencia del alelo menor seguida de su proporción.

SNP: polimorfismos de un solo nucleótido; STGD: distrofia macular de Stargardt.

Tabla 2. Frecuencia de genotipos en polimorfismos de un solo nucleótido de TLR4 objetivo

\begin{tabular}{|c|c|c|c|c|c|c|c|c|}
\hline \multirow[t]{2}{*}{ Posición del gen } & \multirow[t]{2}{*}{ SNP } & \multirow{2}{*}{$\begin{array}{l}\text { Posición del } \\
\text { SNP* }\end{array}$} & \multirow{2}{*}{$\begin{array}{l}\text { Ubicación } \\
\text { del SNP }\end{array}$} & \multirow[t]{2}{*}{ Genotipos } & STED & Familiares & Control & \multirow[t]{2}{*}{ p } \\
\hline & & & & & $n=4$ & $n=12$ & $n=109$ & \\
\hline \multirow[t]{15}{*}{$9 q 32-q 33$} & \multirow[t]{3}{*}{ rs4986790 } & \multirow[t]{3}{*}{13843} & \multirow[t]{3}{*}{ Exón } & $A / A$ & $2(0.5)$ & $11(0.92)$ & $106(0.9724)$ & \multirow[t]{3}{*}{0.0048} \\
\hline & & & & $A / G$ & $2(0.5)$ & $1(0.08)$ & $3(0.0275)$ & \\
\hline & & & & $\mathrm{G} / \mathrm{G}$ & $0(0)$ & $0(0)$ & $0(0)$ & \\
\hline & \multirow[t]{3}{*}{ rs1927911 } & \multirow[t]{3}{*}{8595} & \multirow[t]{3}{*}{ Intrón } & $\mathrm{G} / \mathrm{G}$ & $2(0.5)$ & $6(0.5)$ & $64(0.587)$ & \multirow[t]{3}{*}{0.55} \\
\hline & & & & $A / G$ & $2(0.5)$ & $6(0.5)$ & $34(0.312)$ & \\
\hline & & & & $\mathrm{A} / \mathrm{A}$ & 0 & $0(0)$ & $11(0.101)$ & \\
\hline & \multirow[t]{3}{*}{ rs12377632 } & \multirow[t]{3}{*}{11271} & \multirow[t]{3}{*}{ Intrón } & $T / T$ & $2(0.5)$ & $7(0.58)$ & $31(0.284)$ & \multirow[t]{3}{*}{0.29} \\
\hline & & & & $\mathrm{C} / \mathrm{T}$ & $2(0.5)$ & $5(0.42)$ & $51(0.468)$ & \\
\hline & & & & $\mathrm{C} / \mathrm{C}$ & 0 & $0(0)$ & $27(0.248)$ & \\
\hline & \multirow[t]{3}{*}{ rs2149356 } & \multirow[t]{3}{*}{12740} & \multirow[t]{3}{*}{ Intrón } & $\mathrm{G} / \mathrm{G}$ & $1(0.25)$ & $6(0.5)$ & $61(0.56)$ & \multirow[t]{3}{*}{0.44} \\
\hline & & & & $T / G$ & $2(0.5)$ & $5(0.42)$ & $36(0.33)$ & \\
\hline & & & & $T / T$ & $1(0.25)$ & $1(0.08)$ & $12(0.11)$ & \\
\hline & \multirow[t]{3}{*}{ rs11536889 } & \multirow[t]{3}{*}{16672} & \multirow[t]{3}{*}{$3 \times$ UTR } & $\mathrm{G} / \mathrm{G}$ & $4(1)$ & $12(1)$ & $84(0.771)$ & \multirow[t]{3}{*}{0.36} \\
\hline & & & & $C / G$ & $0(0)$ & $0(0)$ & $22(0.202)$ & \\
\hline & & & & $\mathrm{C} / \mathrm{C}$ & $0(0)$ & $0(0)$ & $3(0.028)$ & \\
\hline
\end{tabular}

${ }^{*}$ RefSeqGene, NG_011475.1; ID del gen, TLR4 7099.

SNP: polimorfismo de un solo nucleótido; STGD: distrofia macular de Stargardt; UTR: región no traducida.

en equilibrio de Hardy-Weinberg. La frecuencia del alelo menor de rs4986790 A/G (G) fue significativamente mayor en pacientes con STGD en comparación con los sujetos control ( 25 frente a 1\%; $p=0.0012$ ).

Las frecuencias de genotipo de los SNP analizados se muestran en la tabla 2. El genotipo que porta el alelo menor de rs4986790 (AG) fue más frecuente en los pacientes con STGD (50\%) en comparación con sus familiares y los sujetos control no relacionados (8 y $2.75 \%$, respectivamente), con significación estadística $(p=0.0048)$.
Las frecuencias de alelos y genotipos de los SNP restantes no fueron significativas entre los casos y los sujetos control $(p>0.05)$. Los familiares no afectados de los pacientes con STGD mostraron frecuencias de alelos y genotipos similares a las observadas en los sujetos control.

\section{Análisis de haplotipos}

Los SNP etiqueta se ubicaron en un bloque de haplotipos, y la magnitud del LD entre los loci bialélicos 
Tabla 3. Frecuencias de haplotipos de SNP etiqueta en el gen TLR4 entre los casos y los sujetos control

\begin{tabular}{|c|c|c|c|c|c|c|c|c|c|}
\hline \multicolumn{5}{|c|}{ SNP etiqueta } & \multirow{2}{*}{$\begin{array}{l}\text { Control } \\
n=109\end{array}$} & \multirow{2}{*}{$\begin{array}{l}\text { STGD } \\
n=4\end{array}$} & \multirow{2}{*}{$\begin{array}{c}\text { Familiares } \\
n=12\end{array}$} & \multirow{2}{*}{$\begin{array}{c}\begin{array}{c}\text { Asociación d haplotipos } \\
\text { con Stargardt* }\end{array} \\
\text { OR (IC } 95 \%)\end{array}$} & \multirow[t]{2}{*}{ p } \\
\hline rs 4986790 & rs1927911 & rs2149352 & rs12377632 & rs11536889 & & & & & \\
\hline A & G & G & C & G & 0.3532 & 0.25 & 0.2083 & 1 & \\
\hline$A$ & A & $\mathrm{T}$ & $\mathrm{T}$ & G & 0.2523 & 0.25 & 0.25 & $1.57(0.21-11.71)$ & 0.66 \\
\hline A & G & G & $\mathrm{T}$ & G & 0.2385 & 0.25 & 0.5 & $1.32(0.15-11.30)$ & 0.8 \\
\hline G & G & $\mathrm{T}$ & $\mathrm{T}$ & G & 0.0138 & 0.25 & 0.0417 & $33.84(2.38-481.17)$ & 0.011 \\
\hline
\end{tabular}

* Los valores de OR se calcularon utilizando tablas de frecuencia de dos vías con los grupos control y con Stargardt. IC: intervalo de confianza; OR: cociente de probabilidades; SNP: polimorfismos de un solo nucleótido.

fue muy alta, con una D' $>0.98$ pareada para todos los casos al comparar pacientes con STGD con los sujetos control. Los haplotipos con una frecuencia superior al $1 \%$ se muestran en la tabla 3 . Encontramos un haplotipo de riesgo significativo. Como se muestra en la tabla 3, el haplotipo GGTTG alcanzó el cociente de probabilidad (OR) más alto ( $p=0.011$; OR: 33.84; intervalo de confianza del 95\%: 2.38-481.17). Curiosamente, el alelo menor de rs4986790 AG (G) está presente en el haplotipo de riesgo.

\section{Discusión}

Los TLR son proteínas transmembrana capaces de activar vías de señalización de respuestas inflamatorias e inmunes. Se expresan en el sistema inmune innato, así como en el sistema nervioso central, incluida la retina. Hasta el momento se han identificado 13 TLR (TLR1-TLR13) en humanos ${ }^{9}$. El TLR4 es un receptor transmembrana involucrado en la detección de LPS de bacterias Gram negativas y muchos otros ligandos exógenos o endógenos. Tiene un dominio extracelular (rico en leucina) responsable del reconocimiento, un dominio transmembrana y un dominio intracelular (similar al dominio interleucina [IL]-1) $)^{15}$. Después de la activación, varias proteínas están involucradas en la señalización intracelular: Molécula adaptadora que induce interferón y contiene al dominio TIR (TRIF) y gen 88 de respuesta primaria de diferenciación mieloide (MYD88) ${ }^{20}$. El TLR4, a través del factor nuclear kappa B (NF-B), es capaz de iniciar la producción de citocinas proinflamatorias como IL-1, IL-6 y el factor de necrosis tumoral- $\alpha$ (TNF- $\alpha$ ). Además, al activar el factor regulador 3 de interferón (IFN) (IRF3), inicia la expresión de genes inducibles por IFN e IFN- $\beta^{21}$. Todas las vías mencionadas controlan la supervivencia celular y la apoptosis ${ }^{22}$.

El gen del TLR4 humano muestra varios SNP que se han asociado con una amplia variedad de enfermedades infecciosas y no infecciosas, incluyendo artritis reumatoide $^{23,24}$, asma bronquial ${ }^{25}$, periodontitis crónica ${ }^{22}$, susceptibilidad a aspergilosis pulmonar y tuberculosis ${ }^{26,27}$, cáncer de pulmón ${ }^{28}$ y riesgo de úlcera péptica ${ }^{29}$.

Los SNP del gen TLR4 presumiblemente influyen en la actividad y la función de su producto ${ }^{16}$. Por ejemplo, las variaciones del gen TLR4 rs4986790 y rs4986791 conducen a variantes sin sentido (Asp299Gly y Thr399lle, respectivamente) que alteran el dominio extracelular de la proteína, y afectan la función del TLR4 al disminuir su capacidad de respuesta ${ }^{30,31}$. El mecanismo por el cual estos SNP inducen hiporreactividad aún no se ha aclarado, pero actualmente se sabe que estas mutaciones inducen cambios conformacionales que afectan el plegamiento y la estabilidad de las proteínas, las interacciones con las proteínas mensajeras y la expresión en la superficie celular ${ }^{32}$. Además, se ha demostrado que Asp299Gly induce un reclutamiento deficiente de MYD88 y TRIF ${ }^{33}$.

Como se mencionó anteriormente, el gen del TLR4 se ha asociado con la apoptosis de las células de la retina, incluidas las RGC y las células endoteliales de la retina ${ }^{9-13}$, pero de manera intrigante, los polimorfismos del TLR4 rs4986790 y rs4986791 pueden inducir la apoptosis de las células estrelladas hepáticas activadas al reducir a fosfo-ERK y $\mathrm{Bcl}-2^{34}$.

La apoptosis del EPR ${ }^{6}$ y la degeneración de fotorreceptores $^{7}$ son fenómenos característicos de la STGD. Aunque la fisiopatología de estos procesos se ha establecido con base en las mutaciones del gen ABCA4 y sus efectos en la STGD ${ }^{5-7}$, se debe explorar la contribución de otros genes para descubrir otros mecanismos de lesión en la STGD.

De forma interesante, rs4986790 se ha descrito como un promotor de la apoptosis ${ }^{34}$. Se debe evaluar la importancia de este efecto, pero para la STGD, podría estar relacionado con la activación microglial mediada por el TLR4, como se sugirió en estudios previos ${ }^{8}$. 


\section{Conclusión}

El alelo menor de los SNP rs4986790 y los genotipos que lo portaban se relacionaron con la STGD clínica en una familia. Somos conscientes de que la principal limitación de nuestro estudio es el bajo número de pacientes incluidos, pero consideramos que la evidencia en estos y otros informes respalda que el gen TLR4 y sus polimorfismos deberían considerarse en futuros estudios para determinar si son marcadores moleculares útiles para el diagnóstico, pronóstico o tratamiento de la STGD.

\section{Conflicto de intereses}

Los autores declaran no tener ningún conflicto de intereses. Solo los autores son responsables del contenido y la redacción de este artículo.

\section{Responsabilidades éticas}

Protección de personas y animales. Los autores declaran que los procedimientos seguidos se conformaron a las normas éticas del comité de experimentación humana responsable y de acuerdo con la Asociación Médica Mundial y la Declaración de Helsinki.

Confidencialidad de los datos. Los autores declaran que siguieron los protocolos de su centro de trabajo sobre la publicación de datos de pacientes.

Derecho a la privacidad y consentimiento informado. Los autores obtuvieron el consentimiento informado de los pacientes y/o sujetos referidos en el artículo. Este documento obra en poder del autor de correspondencia.

\section{Bibliografía}

1. Zahid S, Jayasundera T, Rhoades W, Branham K, Khan N, Niziol LM, et al. Clinical phenotypes and prognostic full-field electroretinographic findings in stargardt disease. Am J Ophthalmol. 2013;155:465-73000.

2. Sohrab MA, Allikmets R, Guarnaccia MM, Smith RT. Preimplantation genetic diagnosis for stargardt disease. Am J Ophthalmol. 2010;149:651-500.

3. Heathfield L, Lacerda M, Nossek C, Roberts L, Ramesar RS. Stargardt disease: towards developing a model to predict phenotype. Eur J Hum Genet. 2013;21:1173-6.

4. Berisha F, Feke GT, Aliyeva S, Hirai K, Pfeiffer N, Hirose T, et al. Evaluation of macular abnormalities in stargardt's disease using optical coherence tomography and scanning laser ophthalmoscope microperimetry. Graefes Arch Clin Exp Ophthalmol. 2009;247:303-9.

5. Tanna P, Strauss RW, Fujinami K, Michaelides M. Stargardt disease: clinical features, molecular genetics, animal models and therapeutic options. Br J Ophthalmol. 2017;101:25-30.

6. Sparrow JR, Boulton M. RPE lipofuscin and its role in retinal pathobiology. Exp Eye Res. 2005;80:595-606.

7. Ma H, Thapa A, Morris L, Redmond TM, Baehr W, Ding XQ, et al. Suppressing thyroid hormone signaling preserves cone photoreceptors in mouse models of retinal degeneration. Proc Natl Acad Sci U S A. 2014:111:3602-7.

8. Kohno H, Chen Y, Kevany BM, Pearlman E, Miyagi M, Maeda T, et al. Photoreceptor proteins initiate microglial activation via toll-like receptor 4 in retinal degeneration mediated by all-trans-retinal. J Biol Chem. 2013;288:15326-41.

9. Morzaev D, Nicholson JD, Caspi T, Weiss S, Hochhauser E, Goldenberg-Cohen $\mathrm{N}$, et al. Toll-like receptor-4 knockout mice are more resistant to optic nerve crush damage than wild-type mice. Clin Exp Ophthalmol. 2015;43:655-65.
10. Okamoto T, Ozawa Y, Kamoshita M, Osada H, Toda E, Kurihara T, et al. The neuroprotective effect of rapamycin as a modulator of the mTORNF-אB axis during retinal inflammation. PLoS One. 2016;11:e0146517.

11. Ulbrich $F$, Schallner $N$, Coburn $M$, Loop $T$, Lagrèze WA, Biermann J, et al. Argon inhalation attenuates retinal apoptosis after ischemia/reperfusion injury in a time- and dose-dependent manner in rats. PLoS One. 2014;9:e115984.

12. Hu L, Yang H, Ai M, Jiang S. Inhibition of TLR4 alleviates the inflammation and apoptosis of retinal ganglion cells in high glucose. Graefes Arch Clin Exp Ophthalmol. 2017;255:2199-210.

13. Zhang TH, Huang CM, Gao X, Wang JW, Hao LL, Ji Q, et al. Gastrodin inhibits high glucose-induced human retinal endothelial cell apoptosis by regulating the SIRT1/TLR4/NF-кBp65 signaling pathway. Mol Med Rep. 2018;17:7774-80.

14. Schneider S, Koch W, Hoppmann P, Ubrich R, Kemmner S, Steinlechner E, et al. Association of toll-like receptor 4 polymorphism with age-dependent systolic blood pressure increase in patients with coronary artery disease. Immun Ageing. 2015;12:4.

15. Rallabhandi P, Bell J, Boukhvalova MS, Medvedev A, Lorenz E, Arditi M, et al. Analysis of TLR4 polymorphic variants: new insights into TLR4/MD-2/ CD14 stoichiometry, structure, and signaling. J Immunol. 2006;177:322-32.

16. Ferwerda B, McCall MB, Verheijen K, Kullberg BJ, van der Ven AJ, Van der Meer JW, et al. Functional consequences of toll-like receptor 4 polymorphisms. Mol Med. 2008;14:346-52.

17. Navarro-Partida J, Martinez-Rizo AB, Ramirez-Barrera $P$, Velazquez-Fernandez JB, Mondragon-Jaimes VA, Santos-Garcia A, et al. Association of toll-like receptor 4 single-nucleotide polymorphisms asp299Gly and thr399lle with the risk of primary open angle glaucoma. Graefes Arch Clin Exp Ophthalmol. 2017;255:995-1001.

18. Navarro-Partida J, Alvarado Castillo B, Martinez-Rizo AB, Rosales-Diaz R, Velazquez-Fernandez JB, Santos A, et al. Association of single-nucleotide polymorphisms in non-coding regions of the TLR4 gene with primary open angle glaucoma in a mexican population. Ophthalmic Genet. 2017;38:325-9.

19. Sarli A, Skalidakis I, Velissari A, Koutsandrea C, Stefaniotou M, Petersen MB, et al. Investigation of associations of ARMS2, CD14, and TLR4 gene polymorphisms with wet age-related macular degeneration in a greek population. Clin Ophthalmol. 2017;11:1347-58.

20. Yamamoto M, Sato S, Hemmi H, Hoshino K, Kaisho T, Sanjo H, et al. Role of adaptor TRIF in the myD88-independent toll-like receptor signaling pathway. Science. 2003;301:640-3.

21. Kutikhin AG. Impact of toll-like receptor 4 polymorphisms on risk of cancer. Hum Immunol. 2011;72:193-206.

22. Sellers RM, Payne JB, Yu F, LeVan TD, Walker C, Mikuls TR, et al. TLR4 asp299Gly polymorphism may be protective against chronic periodontitis. J Periodontal Res. 2016;51:203-11.

23. Kuuliala K, Orpana A, Leirisalo-Repo M, Kautiainen H, Hurme M, Hannonen $\mathrm{P}$, et al. Polymorphism at position +896 of the toll-like receptor 4 gene interferes with rapid response to treatment in rheumatoid arthritis. Ann Rheum Dis. 2006;65:1241-3.

24. Radstake TR, Franke B, Hanssen S, Netea MG, Welsing P, Barrera P, et al. The toll-like receptor 4 asp299Gly functional variant is associated with decreased rheumatoid arthritis disease susceptibility but does not influence disease severity and/or outcome. Arthritis Rheum. 2004:50:999-1001.

25. Werner M, Topp R, Wimmer K, Richter K, Bischof W, Wjst M, et al. TLR4 gene variants modify endotoxin effects on asthma. J Allergy Clin Immunol. 2003;112:323-30.

26. Carvalho A, Pasqualotto AC, Pitzurra L, Romani L, Denning DW, Rodrigues $F$, et al. Polymorphisms in toll-like receptor genes and susceptibility to pulmonary aspergillosis. J Infect Dis. 2008;197:618-21.

27. Schurz H, Daya M, Möller M, Hoal EG, Salie M. TLR1, 2, 4, 6 and 9 variants associated with tuberculosis susceptibility: a systematic review and meta-analysis. PLoS One. 2015;10:e0139711.

28. Kurt H, Ozbayer C, Bayramoglu A, Gunes HV, Degirmenci 囚, Oner KS, et al. Determination of the relationship between rs4986790 and rs4986791 variants of TLR4 gene and lung cancer. Inflammation. 2016;39:166-71.

29. Pohjanen VM, Koivurova OP, Huhta $\mathrm{H}$, Helminen O, Mäkinen JM, Karhukorpi JM, et al. Toll-like receptor 4 wild type homozygozity of polymorphisms +896 and +1196 is associated with high gastrin serum levels and peptic ulcer risk. PLoS One. 2015;10:e0131553.

30. Arbour NC, Lorenz E, Schutte BC, Zabner J, Kline JN, Jones M, et al. TLR4 mutations are associated with endotoxin hyporesponsiveness in humans. Nat Genet. 2000;25:187-91.

31. Long H, O'Connor BP, Zemans RL, Zhou X, Yang IV, Schwartz DA, et al. The toll-like receptor 4 polymorphism asp299Gly but not thr399lle influences TLR4 signaling and function. PLoS One. 2014;9:e93550.

32. Ohto U, Yamakawa N, Akashi-Takamura S, Miyake K, Shimizu T. Structural analyses of human toll-like receptor 4 polymorphisms D299G and T399l. J Biol Chem. 2012;287:40611-7.

33. Figueroa L, Xiong Y, Song C, Piao W, Vogel SN, Medvedev AE, et al. The asp299Gly polymorphism alters TLR4 signaling by interfering with recruitment of myD88 and TRIF. J Immunol. 2012;188:4506-15.

34. Guo J, Loke J, Zheng F, Hong F, Yea S, Fukata M, et al. Functional linkage of cirrhosis-predictive single nucleotide polymorphisms of toll-like receptor 4 to hepatic stellate cell responses. Hepatology. 2009;49:960-8. 